\title{
Photophysics of Caffeic, Ferulic and Sinapic Acids with $\alpha$ - and $\beta$-Cyclodextrins: Spectral and Molecular Modeling Studies
}

\author{
N. Rajendiran*, T. Mohandoss and J. Thulasidhasan \\ Department of Chemistry, Annamalai University, Annamalai nagar - 608002, Tamilnadu, India \\ *drrajendiran@rediffmail.com, Phone: +91 9486628800.
}

Keywords: Hydroxycinnamic acids; Cyclodextrins; Inclusion complex; Intramolecular charge transfer; Molecular modeling.

\begin{abstract}
Encapsulation of caffeic acid (CAA), ferulic acid (FEA) and sinapic acid (SIA) molecules with $\alpha-C D$ and $\beta-C D$ was studied by UV-visible, fluorescence, time-resolved fluorescence and molecular modelling techniques. This analysis reports the dual emission properties of the above hydroxycinnamic acids (HCAs) in the aqueous cyclodextrin (CD) solution. The shorter wavelength bands originated from the locally excited state and the longer wavelength bands due to the emission from an intramolecular charge transfer (ICT) state. The ratio of the TICT emission to the normal emission increased along with $\alpha-C D$ and $\beta-C D$ concentration. The guest:host inclusion complexation studies indicates (i) HCAs forms 1:1 inclusion complex and (ii) acrylic group present in the interior part of the $\mathrm{CD}$ cavity and $\mathrm{OH} / \mathrm{OCH}_{3}$ groups present in the upper part of the $\mathrm{CD}$ cavity. $\mathrm{pH}$ studies suggest proton transfer reactions follow the same trend in these molecules. PM3 optimizations were also carried out to assign the encapsulation of the HCA molecules.
\end{abstract}

\section{Introduction}

Cyclodextrins (CD) are cyclic oligosaccharides that form hydrophobic and restrictive cavities with hydrophilic external walls in aqueous solution, given those attractive dissimilar microenvironments with a capability to include guest molecules. The interesting and exciting phenomenon of intramolecular charge transfer (ICT) in many drugs and dyes [1-10] has engendered a deep concentration in studying its photophysical properties in hydrophobic cavities of cyclodextrins. The dependence of ICT emission on environmental polarity, viscosity as well as rotational mobility of donor moiety makes these drugs and dyes molecules outstanding candidates [10-18] for examining microscopic molecular environments. Some guest molecules attribute the change in ICT emission to reduced polarity effect inside the cavity while others stress that the structure of inclusion complex and CD cavity constraint has a greater influence on ICT process than the local polarity. As the formation of ICT involves both rotations of donor moiety and micropolar environments the overall effect of $\mathrm{CD}$ encapsulation on photophysics of molecules seems to be complicated.

In our earlier communications, we reported the dual emission characteristics of many drugs and dyes [1-7] found that ICT and hydrogen bonding plays an important role in polar solvents and $\mathrm{CD}$. The drug and dye molecules interestingly forms inclusion complex with cyclodextrin having a preferential orientation of the $\mathrm{OH} /$ methoxy group sticking outside in aqueous solvent and just the reverse is the case in nonaqueous solvent. We have been intrigued by this observation of preferential orientation and wanted to investigate the orientation and structure of $\alpha-C D$ and $\beta-C D$ inclusion complex of these three HCA molecules. The driving force and the affinity of hydrogen bonding may not act similarly in a smaller cavity. This paper concerns the guest-host inclusion complex studies of $\alpha-\mathrm{CD}$ and $\beta$-CD with caffeic acid (CAA, 3,4-dihydroxy-cinnamic acid), ferulic acid (FEA, 4-hydroxy-3-methoxycinnamic acid) and sinapic acid (SIA, 4-hydroxy-3,5dimethoxycinnamic acid) (Fig. 1). 


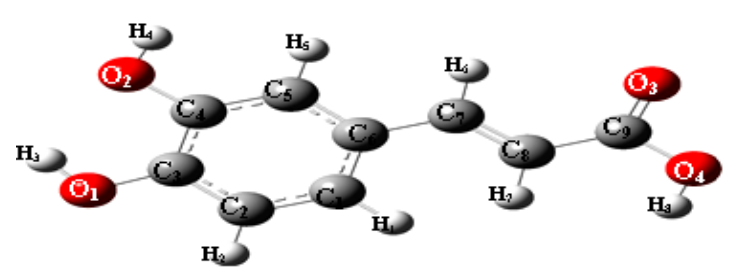

(a)

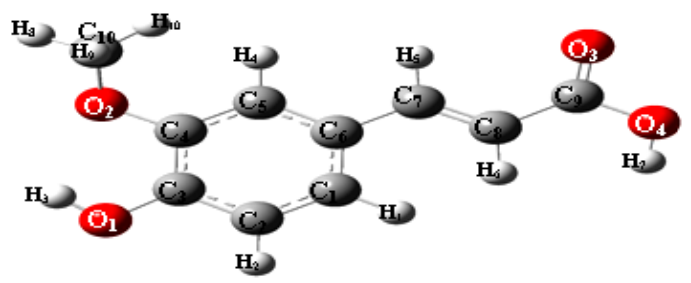

(b)

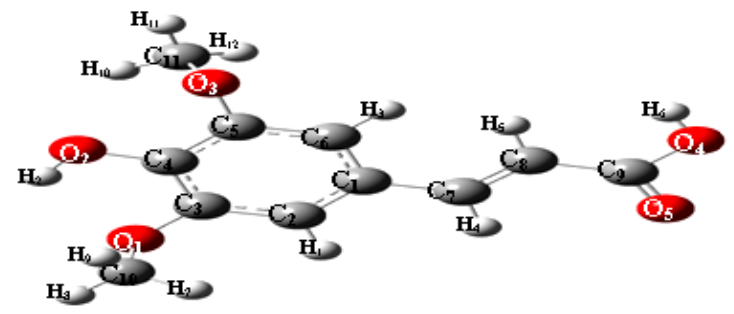

(c)

Figure 1. PM3 optimized structures of (a) CAA, (b) FEA and (c) SIA.

CAA, FEA and SIA are consists of both phenolic and acrylic functional groups. They are found in all plants because it is a key intermediate in the biosynthesis of lignin, one of the principal components of plant biomass and its residues [19]. These acids have a variety of potential pharmacological effects in vitro studies; i.e., antioxidant in vitro and also in vivo, inhibitory effect on cancer cell proliferation by oxidative mechanism [20], immunomodulatory and anti-inflammatory activity, inhibits carcinogenesis, significant decrease in growth of colon tumors [21], antibacterial [22], antioxidant [23], antitumor activity against breast cancer [24] and liver cancer [25]. Because of these importance of these acids, in this paper we studied the effect of $\alpha-C D$ and $\beta-C D$ of caffeic acid (CAA), ferulic acid (FEA), and sinapic acid (SIA) were examined and the results are compared (Fig. 1) by UV-visible, emission and PM3 methods.

\section{Experimental section}

\subsection{Materials}

CAA, FEA, SIA (hydroxy cinnamic acids, HCAs), $\alpha-\mathrm{CD}$ and $\beta$-CD were purchased from Sigma-Aldrich chemical company and used without further purification. The purity of the compounds was checked for similar fluorescence spectra when excited with different wavelengths.

\subsection{Preparation of CD Solution}

The concentration of stock solution of the HCAs was $1 \times 10-3 \mathrm{M}$. The stock solution $(0.2 \mathrm{ml})$ was transferred into $10 \mathrm{ml}$ volumetric flasks. To this, varying concentration of CD solution $(1 \times 10-3$ to $1 \times 10-2 \mathrm{M})$ was added. The mixed solution was diluted to $10 \mathrm{ml}$ with triply distilled water and shaken thoroughly. The final concentration of guest molecules in all the flasks was $2 \times 10-5 \mathrm{M}$. The experiments were carried out at room temperature at $300 \mathrm{~K}$.

\subsection{Instruments}

Absorption spectral measurements were carried out with a Shimadzu (model UV 2600) UV-visible spectrophotometer and steady-state fluorescence measurements were made by using a 
Shimadzu spectrofluorimeter (model RF-5301). The excitation and emission slit widths for all the experiments are $10 \mathrm{~nm}$. The fluorescence lifetime measurements were performed using a picoseconds laser and single photon counting setup from Jobin-Vyon IBH (Madras University, Chennai). $\mathrm{pH}$ of the solution was measured in a Elico $\mathrm{pH}$ meter model LI-120.

\subsection{Molecular modeling studies}

The theoretical calculations were executed with Gaussian 09W package. The ground state geometry of the CAA, FEA, SIA, $\alpha-C D$ and $\beta-C D$ were builded with the help of Spartan 08 and then optimized by semiempirical PM3 method. CD was completely optimized by PM3 without any symmetry restriction. The glycosidic oxygen atoms of $\mathrm{CD}$ were placed onto the $\mathrm{XY}$ plane and their centre was defined as the centre of the coordination system. The primary hydroxyl groups were placed pointing toward the positive $\mathrm{Z}$ axis. The inclusion complex was constructed from the PM3 optimized CD and guest molecules. The longer dimension of the guest molecule was initially placed onto the $\mathrm{Z}$ axis. The position of the guest was determined by the $\mathrm{Z}$ coordinate of one selected atom of the guest. The inclusion process was simulated by putting the guest on one end of $C D$ and then letting it pass through the CD cavities. Since the semiempirical PM3 method has been proved to be a powerful tool in the conformational study of CD inclusion complexes and has high computational efficiency, we selected semiempirical PM3 method to study the inclusion process of CDs with HCAs.

\section{Results and discussion}

\subsection{Absorption and fluorescence spectral studies}

Fig. 2-4 shows the absorption and emission spectra of CAA, FEA, and SIA, in aqueous solution containing varying concentration of $\alpha-C D$ and $\beta-C D$ keeping the concentration of the guest molecules fixed $\left(2 \times 10^{-5} \mathrm{M}\right)$. The inset Fig. 2-4 depict the changes for the absorbance and fluorescence intensities observed as a function of the $\alpha-\mathrm{CD}$ and $\beta-\mathrm{CD}$ concentrations added. With addition of $\alpha-C D$ and $\beta-C D$ the absorption maxima shifts to longer wavelength with a gradual change in absorbance. All the HCA (CAA, FEA and SIA) molecules give structured absorption spectra. This shows that the hydroxy and methoxy groups are not effectively interacting much with the aromatic ring in the ground state. The above three HCA compounds can be considered as the derivatives of the cinnamic acid/ para hydroxycinnamic acid. In aqueous solutions, the absorption and emission maxima of the above HCAs are largely red shifted than that of cinnamic acid and para hydroxy cinnamic acid (CA: $\lambda_{\text {abs }} \sim 275,221 \mathrm{~nm}, \lambda_{\text {flu }} \sim 321,301 \mathrm{~nm}$; para hydroxy cinnamic acid: $\lambda_{\text {abs }}$ $\left.\sim 285,218 \mathrm{~nm}, \lambda_{\text {flu }} \sim 425,326 \mathrm{~nm}\right)$.

Upon increasing the $\alpha-C D$ concentration, the absorption intensities of the HCAs are to some extent increased and the absorption maxima are slightly red shifted whereas in $\beta-\mathrm{CD}$ it is largely red shifted (CAA: $\alpha-\mathrm{CD}-\lambda_{\text {abs }} \sim 313$ to $316 \mathrm{~nm}, \beta-\mathrm{CD}-\lambda_{\text {abs }} \sim 313$ to $330 \mathrm{~nm}$; FEA: $\alpha-\mathrm{CD}$ $\lambda_{\mathrm{abs}} \sim 310$ to $313 \mathrm{~nm}, \beta-\mathrm{CD}-\lambda_{\mathrm{abs}} \sim 310$ to $322 \mathrm{~nm}$; SIA: $\alpha-\mathrm{CD}-\lambda_{\mathrm{abs}} \sim 308$ to $314 \mathrm{~nm}, \beta-\mathrm{CD}-\lambda_{\mathrm{abs}}$ $\sim 308$ to $320 \mathrm{~nm}$ ). With addition of $\alpha-\mathrm{CD}$ and $\beta-\mathrm{CD}$, the increase in absorbance along with red shift indicating the formation of guest: $\mathrm{CD}$ inclusion complex. 

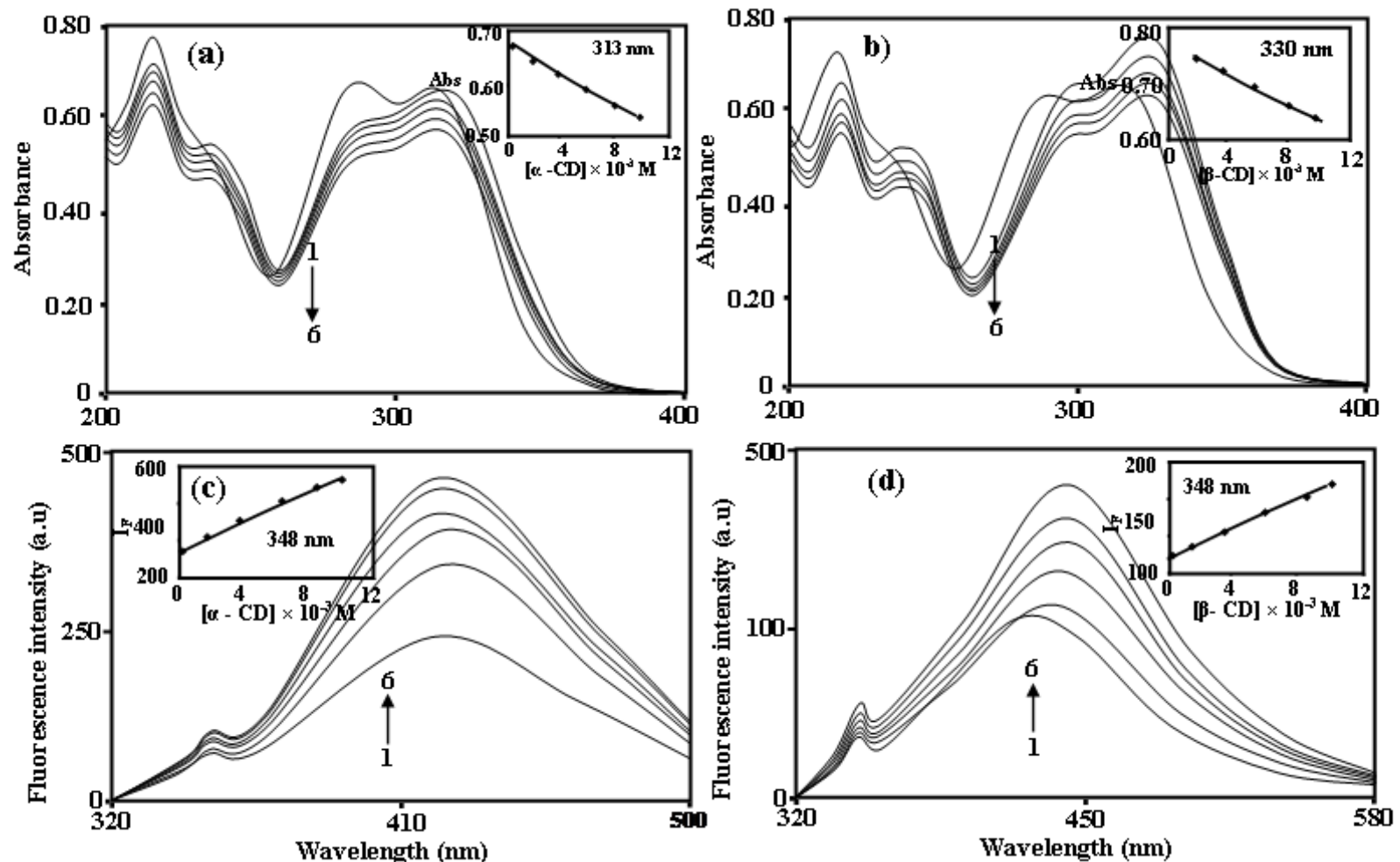

Figure 2. Absorbance and fluorescence spectra of CAA in different $\alpha-C D$ and $\beta$-CD concentrations (M): 1) 0, 2) 0.001, 3) 0.002, 4) 0.004, 5) 0.006, 6) 0.008, 7) 0.01. Insert figure Absorbance and fluorescence intensity vs. CD concentrations.
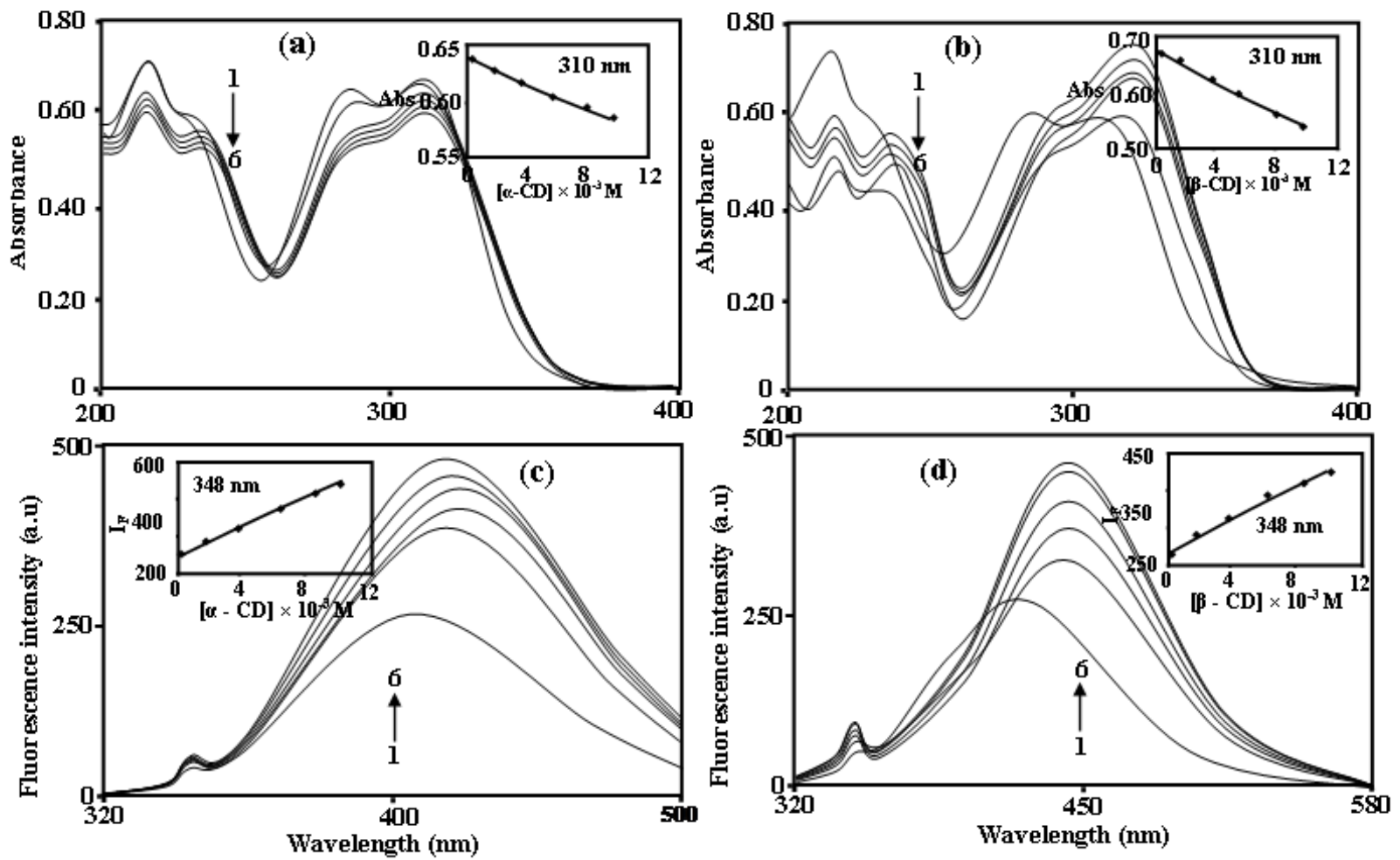

Figure 3. Absorbance and fluorescence spectra of FEA in different $\alpha-C D$ and $\beta$-CD concentrations (M): 1) 0, 2) 0.001, 3) 0.002, 4) 0.004, 5) 0.006, 6) 0.008, 7) 0.01. Insert figure Absorbance and fluorescence intensity vs. CD concentrations. 

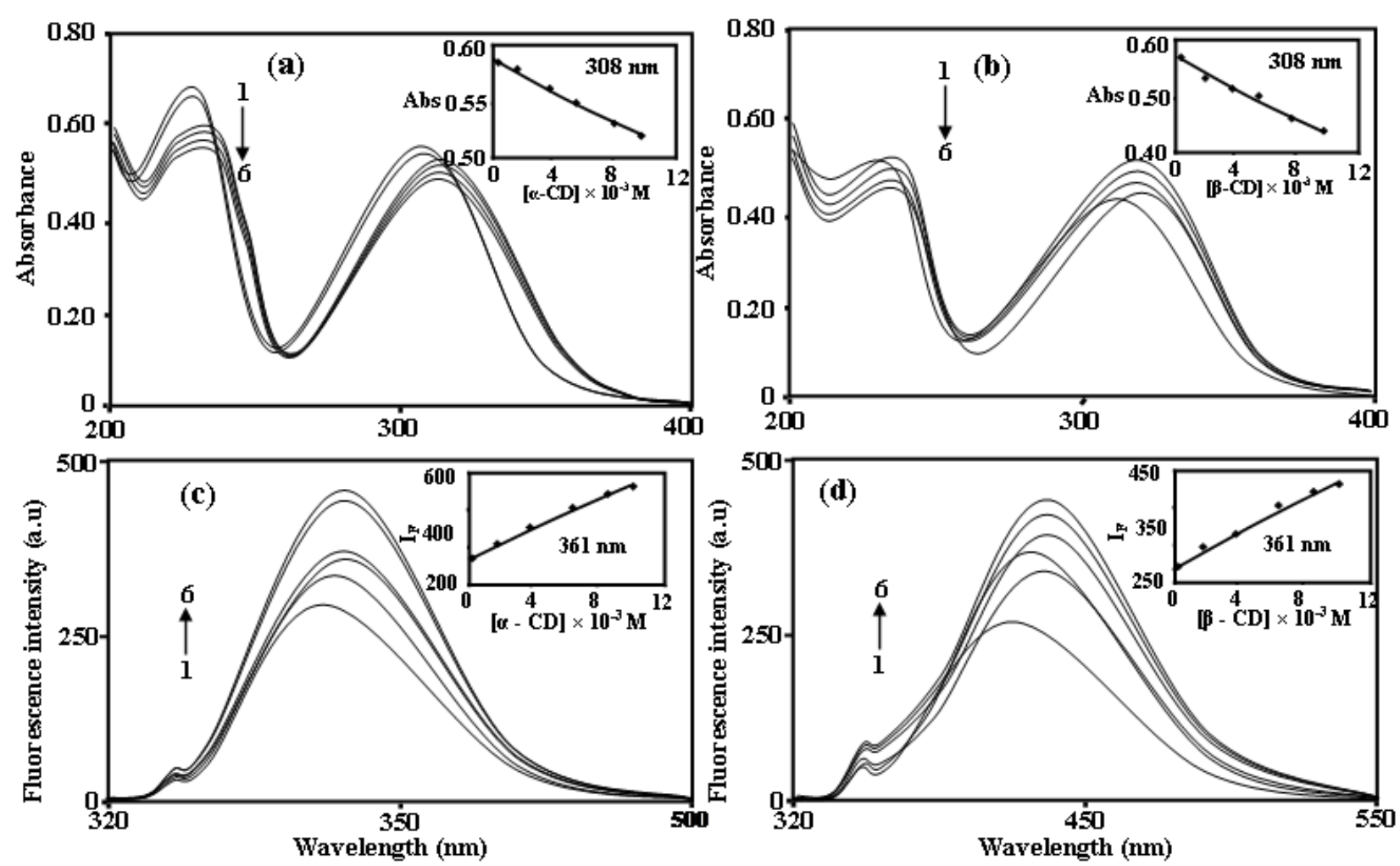

Figure 4. Absorbance and fluorescence spectra of SIA in different $\alpha-C D$ and $\beta$-CD concentrations $(\mathrm{M}):$ 1) 0 , 2) $0.001,3) 0.002$, 4) 0.004 , 5) 0.006, 6) 0.008, 7) 0.01. Insert figure Absorbance and fluorescence intensity vs. CD concentrations.

The above results showed that, the guest molecules were transferred from more protic environments (bulk aqueous phase) to less protic CD cavity environments [1-10, 26-31]. Similar spectral shifts of all the HCAs showed that same type of the functional group is encapsulated and this functional group is interacted with the secondary hydroxyl groups of the CD.

The appearance of isosbestic point indicates the formation of a 1:1 host-guest type inclusion complex between the CDs and HCA molecules. For the guest:CD inclusion complexes, determination of the formation constant and the stoichiometry are important. Both parameters are calculated with the help of the Benesi-Hildebrand equation [32]. The formation constant $(K)$ and stoichiometric ratio of the inclusion complex of the guest (HCAs) can be determined from the Benesi-Hildebrand relation assuming the formation of a 1:1 HCA: CD complex is

$$
1 / \Delta \mathrm{A}=1 / \Delta \mathrm{E}+1 / K[\mathrm{HCA}]_{0} \Delta \mathrm{E} \cdot[\mathrm{CD}]_{0}
$$

where $\Delta \mathrm{A}$ is the difference between the absorbance of HCA in the presence and absence of $\mathrm{CD}, \Delta \mathrm{E}$ is the difference between the molar absorption coefficient of $\mathrm{CD}$ and the inclusion complex, $[\mathrm{HCA}]_{0}$ and $[\mathrm{CD}]_{0}$ are the initial concentration of HCA and $\mathrm{CD}$ respectively.

Fig. 5 depicts a plot of $1 / \Delta \mathrm{A}$ as a function of $1 /[\mathrm{CD}]$ for HCA molecule. Good correlations were obtained confirming the formation of a 1:1 complex, the $\mathrm{K}$ value is calculated from the intercept and slope of the plot (binding constant value, unit- $\mathrm{M}^{-1}$ : CAA: $\boldsymbol{\alpha}-\mathbf{C D} \sim$ abs -288 , flu-387, CAA: $\boldsymbol{\beta}-\mathbf{C D} \sim$ abs -363, flu -431; FEA: $\boldsymbol{\alpha}-\mathbf{C D} \sim$ abs -249 , flu- 479, FEA: $\boldsymbol{\beta}$-CD $\sim$ abs -541 , flu -625 M; SIA: $\boldsymbol{\alpha}-\mathbf{C D} \sim$ abs -413 , flu-666, SIA: $\boldsymbol{\beta}-\mathbf{C D} \sim$ abs -252 , flu -454). Fig. 5 shows the double reciprocal plot for complexation of HCAs with the CDs, which confirms 1:1 complexation formed between the host and guest molecules in the ground and excited state.

$\left(\Delta \mathrm{G}\right.$ (-ve value) unit- $\mathrm{kcal} \mathrm{mol}^{-1}: \mathbf{C A A}: \boldsymbol{\alpha}-\mathbf{C D} \sim$ abs -2.95 , flu-3.14, CAA: $\boldsymbol{\beta}-\mathbf{C D} \sim$ abs -3.30 , flu -3.49; FEA: $\boldsymbol{\alpha}-\mathbf{C D} \sim$ abs -3.12 , flu- 3.93, FEA: $\boldsymbol{\beta}-\mathbf{C D} \sim$ abs -3.51 , flu -3.89 ; SIA: $\boldsymbol{\alpha}-\mathbf{C D} \sim$ abs - 3.62, flu-3.91, SIA: $\boldsymbol{\beta}-\mathbf{C D} \sim$ abs -3.12, flu -3.68).

The reciprocal of the absorbance/fluorescence intensity of the inclusion complex versus the reciprocal of $\mathrm{CD}$ concentration is excellent, because the formation constant and stoichiometry can 
be simultaneously obtained from the plot. Benesi-Hildebrand relation [26-31] should give a straight indicates 1:1 inclusion complex to be formed in the ground and excited state.
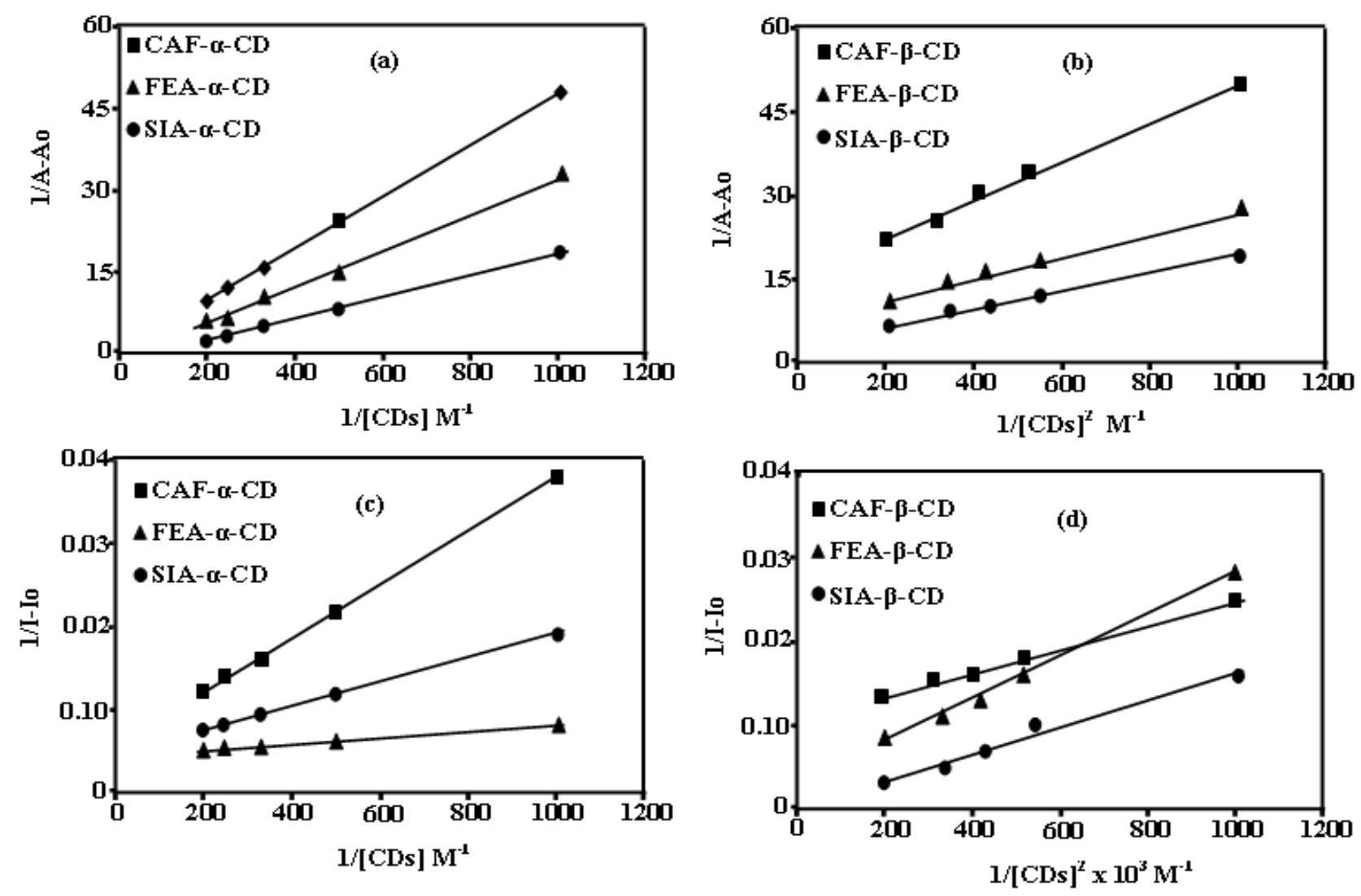

Figure 5. Benesi-Hildebrand plot for 1:1 inclusion complexation of CAF, FEA, and SIA with $\alpha-\mathrm{CD}$ and $\beta-\mathrm{CD}$ at $p \mathrm{H} \sim 7$ : (a) and (b) plot of $1 / \mathrm{A}-\mathrm{A}_{\mathrm{o}}$ vs. $1 /[\mathrm{CD}]$, (c) and (d) plot of 1/I- $\mathrm{I}_{\mathrm{o}} \mathrm{vs.} 1 /[\mathrm{CD}]$.

\subsection{Emission spectra}

Fig 2-4 depicts the emission spectra of HCAs (excited at $300 \mathrm{~nm}$ ) with varying concentration of $\alpha-C D$ and $\beta-C D$. The CD free aqueous solution of the HCAs shows dual fluorescence, one around $\sim 345 \mathrm{~nm}$ (shorter wavelength or normal band) with very low emission intensity and another band around 420 - $445 \mathrm{~nm}$ (longer wavelength or ICT band) with slightly higher emission intensity of the HCAs. With an addition of both CDs, the shorter wavelength (SW) and longer wavelength (LW) emission intensities increases, however, the rate of enhancement of the LW emission intensity is greater than that for the SW emission wavelength. That is, with addition of $\alpha-C D$ and $\beta-C D$, the SW fluorescence intensity shows a little enhancement and the wavelength maximum was not significantly changed. However, the longer emission maximum shows a large enhancement with a red shift. The red shift as well as enhancement of the emission intensity of the LW band could be observed only when the concentration of $\alpha-\mathrm{CD}$ and $\beta$-CD increased (Fig. 2-4).

In the HCA molecules, the enhancement of the fluorescence intensity is due to the formation of a HCA-CD inclusion complex. In the inclusion complex, HCA molecule is encapsulated in the non polar part of the CD cavity. Thus, in the HCA molecules, with an increase in the CD concentration the red shift is observed in the emission maximum (Fig. 2 - 4). The increase in the emission intensity and band width of the HCA molecule in the CD solutions suggest the formation of a 1:1 inclusion complex. As like in absorption, the formation constant value is determined from the slope and the intercept of the plot (Fig. 5). This formation constant value in the emission is greater than that obtained from the absorption, because the change in emission intensity upon addition of $\mathrm{CD}$ is significantly greater than the absorption. A significant raise in the LW emission intensity compared with the nearly constant absorbance or slight increase in SW suggest that intramolecular charge transfer (ICT) emission is increased in the presence of CD (Fig. 2 - 4). For example, in the presence of $1 \times 10^{-2} \mathrm{M} \mathrm{CD}$, the emission intensity of HCA is two times greater than that in the absence 
of CD. It should be noted that such a comparison is significant because of the small shift in the fluorescence and absorption spectra. A similar characteristic is also observed in various guests by various authors [1-18].

Considering the length of HCA molecules and the CD cavities, one can expect two types of inclusion complex may be formed. The first type (type-1), $\mathrm{OH} / \mathrm{OCH}_{3}$ group is projected towards the larger rim of the CD cavity representing a slightly polar and slightly rigid environment and the second type (type-2), the acrylic group of the HCAs are headed towards the smaller rim of the CD cavity representing a less polar and most rigid environment.

Let us consider the type- 1 arrangement, the carbonyl group of HCAs are entrapped within the CD cavity, hence the HCA molecules are not form a hydrogen bond with the bulk aqueous solution. In that case, the ICT state formation energy barrier increases due to the relatively less polar environment inside the CD cavity compared to water as a solvent. These will help an enhancement of the LW band but at the same time the SW will be less stabilized by solvation due to comparatively less polar environment inside the CD cavity causing an increase in the energy gap between the ICT state and low lying singlet state. Thus, the non-radiative decay rate from the ICT state increases causing an increase in the ICT intensity along with a red shift. The non polar CD cavity is capable of stabilizing the ICT state of HCA a lot by increasing its energy level by preventing the hydrogen bond with water and the carbonyl group of the guest. This will cause a decrease in non-radiative decay of ICT state and a increase in ICT emission band intensity.

In type-2 encapsulation, the cavity will impose a restriction about the free rotation of the $\mathrm{OH} / \mathrm{OCH}_{3}$ group in the excited state and the $\mathrm{HCA}$ molecule will face non polar environment causing a decrease in rate of charge transfer and thereby an enhancement of the normal emission (SW) band. For this type of complex carbonyl group making a hydrogen bond with the aqueous solution is very much possible as the carbonyl group is available in the bulk solution. Hence, the ICT emission intensity should be almost unaltered because the energy barrier to form ICT state not only depends upon rotation of $\mathrm{OH} / \mathrm{OCH}_{3}$ but also on hydrogen bonding between the guest and the water. The energy barrier for formation of ICT state does not change as long as hydrogen bonding is still possible [33-35] between HCA and water molecules. So the huge enhancement of the ICT state suggests that among these two types of orientations of HCA molecule inside the CD cavity results in a huge observed enhancement of the LW band with red band shift as it is emitted from the most nonpolar state of HCA and a small enhancement of the SW state without any spectral shift.

We know that the ICT state arises from the mutual orthogonal position of the two $\pi$-moieties, which transition is forbidden. The hydrogen bond formation of type-1 inclusion complex with water molecules is not possible because the $\mathrm{C}=\mathrm{O}$ group is encapsulated in the $\mathrm{CD}$ cavity, which is not takes part in hydrogen bond formation. In type- 2 inclusion complex, the $\mathrm{OH} / \mathrm{OCH}_{3}$ group faces a hindrance of its free rotation in the excited state of HCA, the energy barrier for formation of the ICT state does not change as long as hydrogen bonding is still possible between $\mathrm{C}=\mathrm{O}$ and water molecules. So the SW band remains unaffected. When two $\pi$-moieties of the HCA are not in orthogonal position inside the CD cavity it causes an enhancement of radiative transition probability [33-35] of the ICT state and an increase in the ICT band. But the hydrogen bond formation of the $\mathrm{C}=\mathrm{O}$ group of HCA with water molecules makes the ICT state more stable by lowering its energy level resulting in an extra red shift of the low energy (ICT) band. The observed feature when the excitation wavelength is decreased below $300 \mathrm{~nm}$, the SW band intensity remains unaltered whereas the ICT band shows an enhancement along with a red shift. The gradual change in relative intensity and position of ICT bands imply that type 1 complex is more favoured than type -2 complex. In view of above discussion one can easily conclude that type 1 complexes are more favoured in the HCA molecules.

\subsection{Fluorescence lifetime}

Time resolved analysis of fluorescence decay shows that both drugs molecules were give biexponential in water as well as emission at $300 \mathrm{~nm} \mathrm{CD}$ solutions. Table 1 summarizes the life time data and decay profile of the CAA, FEA and SIA drugs in aqueous and CD mediums, the 
average life time $<\tau>$ was calculated using the equation (2) where $\tau$ is lifetimes of the component; a is the corresponding pre-exponential factors, and $t$ is the time.

$$
\langle\tau\rangle=\sum \tau_{i} a_{i}
$$

In aqueous solution, with addition of $\mathrm{CD}$, the decay time of the SW band does not changed but the decay time of ICT band increases which suggests the formation of HCA/CD inclusion complex. In the absence of CDs (or in aqueous medium) the life times to be $0.15 \mathrm{~ns}$ and $0.30 \mathrm{~ns}$ for SW and ICT state respectively for HCA molecules which were less than that of the CD medium. The decay of $300 \mathrm{~nm}$ was not significantly different to $\alpha-C D$ and $\beta-C D$ concentrations. The decay time of the ICT component increases significantly from water to CD solutions while no significant change observed in the SW component of CAA, FEA and SIA molecules. The ICT emission decay in the water and CD exhibits a slow decay as a major decay component while normal emission is observed very fast. These decay behavior indicates in the presence of CD the HCA molecules ICT state is increased. The decay times of the ICT were higher than that of normal emission within experimental uncertainty. This indicated that the equilibrium was not achieved between the normal and the ICT states in water in a rather short period. However, in the presence of CD, the equilibrium between the normal and the ICT states was modified by the formation of the CD inclusion complexes. Thus, the above results confirm the formation of the ICT become more favorable in the CD solution compared than in the water.

Table 1. Fluorescence decay profile of CAA, FEA and SIA with different $\alpha-C D$ and $\beta-C D$ concentrations.

\begin{tabular}{|l|l|l|l|l|l|l|}
\hline Concentration of CD (M) & CAA & \multicolumn{1}{l}{ FEA } & SIA & \\
\hline & $\boldsymbol{\alpha}-C D$ & $\boldsymbol{\beta}$-CD & $\boldsymbol{\alpha}$-CD & $\boldsymbol{\beta}$-CD & $\boldsymbol{\alpha}$-CD & $\boldsymbol{\beta}$-CD \\
\hline & $\tau$ & $\tau$ & $\tau$ & $\tau$ & $\tau$ & $\tau$ \\
\hline 0 (without CD) & 0.15 & 0.15 & 0.16 & 0.16 & 0.15 & 0.15 \\
& 0.30 & 0.30 & 0.31 & 0.31 & 0.30 & 0.30 \\
\hline 0.002 & 0.15 & 0.15 & 0.16 & 0.16 & 0.15 & 0.15 \\
& 0.32 & 0.32 & 0.31 & 0.21 & 0.31 & 0.31 \\
\hline 0.004 & 0.16 & 0.16 & 0.16 & 0.16 & 0.16 & 0.16 \\
& 0.32 & 0.32 & 0.33 & 0.23 & 0.33 & 0.33 \\
\hline 0.006 & 0.16 & 0.16 & 0.17 & 0.17 & 0.16 & 0.16 \\
& 0.33 & 0.33 & 0.35 & 0.35 & 0.33 & 0.33 \\
\hline 0.008 & 0.16 & 0.16 & 0.17 & 0.17 & 0.17 & 0.17 \\
& 0.34 & 0.34 & 0.36 & 0.36 & 0.34 & 0.34 \\
\hline 0.010 & 0.16 & 0.16 & 0.17 & 0.18 & 0.17 & 0.17 \\
& 0.35 & 0.35 & 0.36 & 0.36 & 0.34 & 0.34 \\
\hline
\end{tabular}

\subsection{Effect of pH}

The absorption and fluorescence spectra of HCA molecules have been studied in the $\mathrm{H} 0 / \mathrm{pH} / \mathrm{H}$ - range of -1 to 11 . The relevant data are given in Table 2 . With an increase in $\mathrm{pH}$ from 2.0 , around $\mathrm{pH} 4.0$ the absorption and emission maximum is blue shifted. The blue shift in the absorption and emission spectra below $\mathrm{pH} \sim 5$ confirms that deprotonation takes place at the carboxyl group. Further increase the $\mathrm{pH}$ from 5, HCA molecules gives a newly blue shifted spectrum with the maximum. This suggests that formation of a dianion obtained by the deprotonation of the $\mathrm{OH}$ group. It is well known fact that deprotonation of $\mathrm{COOH}$ group gives blue shifted absorption and emission maxima whereas deprotonation of hydroxyl group gives red shifted absorption and emission maxima [27-31]. In the HCA molecules, unusual blue shift noticed in the hydroxyl anion indicating that carboxy monoanion decrease the interaction between the ionic substituent and the aromatic ring [26-31]. 
Table 2. Various prototropic (absorption and fluorescence) maxima of CAA, FEA and SIA in aqueous and $\alpha-C D, \beta-C D$ medium.

\begin{tabular}{|c|c|c|c|c|c|c|c|c|c|c|c|c|c|c|c|c|c|c|}
\hline \multirow[t]{3}{*}{ Species } & \multicolumn{6}{|c|}{ CAA } & \multicolumn{6}{|c|}{ FEA } & \multicolumn{6}{|c|}{ SIA } \\
\hline & \multicolumn{2}{|c|}{ Aqueous } & \multicolumn{2}{|c|}{$\alpha-C D$} & \multicolumn{2}{|c|}{$\beta-C D$} & \multicolumn{2}{|c|}{ Aqueous } & \multicolumn{2}{|c|}{$\alpha-C D$} & \multicolumn{2}{|c|}{$\beta-C D$} & \multicolumn{2}{|c|}{ Aqueous } & \multicolumn{2}{|c|}{$\alpha-C D$} & \multicolumn{2}{|c|}{$\beta-C D$} \\
\hline & $\lambda_{\mathrm{abs}}$ & $\lambda_{\mathrm{flu}}$ & $\lambda_{\text {abs }}$ & $\lambda_{\text {flu }}$ & $\lambda_{\mathrm{abs}}$ & $\lambda_{\text {flu }}$ & $\lambda_{\text {abs }}$ & $\lambda_{\text {flu }}$ & $\lambda_{\text {abs }}$ & $\lambda_{\text {flu }}$ & $\lambda_{\mathrm{abs}}$ & $\lambda_{\text {flu }}$ & $\lambda_{\mathrm{abs}}$ & $\lambda_{\text {flu }}$ & $\lambda_{\text {abs }}$ & $\lambda_{\text {flu }}$ & $\lambda_{\text {abs }}$ & $\lambda_{\text {flu }}$ \\
\hline \multirow[t]{3}{*}{ Neutral } & 322 & 445 & 325 & 436 & 325 & 441 & 322 & 452 & 325 & 436 & 321 & 444 & 320 & 460 & 327 & 442 & 322 & 446 \\
\hline & 232 & 362 & 243 & 361 & 242 & 360 & 295 & 361 & 243 & 361 & 235 & 350 & 234 & 360 & 242 & 361 & 235 & 362 \\
\hline & 217 & & 215 & & 218 & & $\begin{array}{l}233 \\
217\end{array}$ & & 217 & & & sh & & & 221 & & & \\
\hline \multirow{3}{*}{$\begin{array}{l}\text { Mono- } \\
\text { anion }\end{array}$} & 316 & 429 & 309 & 418 & 325 & 443 & 314 & 438 & 308 & 439 & 322 & 444 & 312 & 442 & 304 & 445 & 323 & 444 \\
\hline & 291 & 363 & 286 & 361 & 242 & 360 & 288 & $363 \mathrm{sh}$ & 287 & & 234 & 350 & 230 & 361 & 226 & 362 & 233 & 362 \\
\hline & 215 & & 215 & & 219 & sh & $\begin{array}{l}228 \\
216\end{array}$ & & 214 & & 215 & sh & & & & & & \\
\hline \multirow[t]{3}{*}{ Dianion } & 310 & 424 & 310 & 450 & 324 & 443 & 310 & 423 & 307 & 414 & 322 & 444 & 308 & 442 & 304 & 442 & 321 & 446 \\
\hline & 287 & 362 & 287 & 363 & 234 & 360 & 287 & 363 & 287 & & 236 & 350 & 220 & 362 & 227 & 361 & 232 & 362 \\
\hline & 215 & sh & 214 & & 217 & sh & 215 & sh & 215 & & 218 & & & & & & & \\
\hline
\end{tabular}

q- Quenching, w - weakly fluorescent

\subsection{Molecular modeling studies}

In order to confom our experimental data and conclude the mode of inclusion, we performed molecular modeling investigation of the process of inclusion of CAA, FEA and SIA within $\alpha-C D$ and $\beta-C D$ cavities by using Gaussian $09 \mathrm{~W}$ software and the minimum energy structure of the complex is shown in Fig. 6 and the energy features were listed out in the Table 3.

From the minimum energy, we predicts the inclusion complexes structure that the guests were involved (CAA, FEA and SIA) in one hydrogen bond listed in Fig. 6, first one with the primary hydroxyl group and the second one is through glycosidic oxygen of $\alpha-C D$ and $\beta-C D$. The lowest energy structure reveals that CAA, FEA and SIA were included along the molecular axis of CDs, but its molecular axis were not exactly perpendicular to the CDs symmetry axis rather it were slightly tilted to allow maximum hydrogen bonding interactions between the host and the guest.

Acrylic group of the CAA, FEA and SIA enters deep into the CDs cavity and approaches the secondary hydroxyl rim of $\alpha-\mathrm{CD}$ and $\beta$-CD. Fig. 6 reveals that both aromatic $-\mathrm{OH} / \mathrm{CH}_{3}$ group and carbonyl group of CAA, FEA and SIA are not form hydrogen bond with CDs and thus van der Waals forces and hydrophobic interactions stabilizes the inclusion complexes. Thus van der Waals forces and hydrophobic interactions are the main driving forces to form the inclusion complex along with the high enthalpy gain. The inner nanocavities of CDs were not absolutely hydrophobic rather its polarity was comparable to that of alcoholic solution [35]. Due to the presence of glycosidic oxygen and secondary and primary hydroxyl groups, CDs can accommodate polar substituent groups and these groups can readily form hydrogen bonds with the polar counterparts of the CDs. In fact it is well known that substituent of aromatic rings capable of $\mathrm{H}$-bonding and it can find the $-\mathrm{OH}$ groups of the CDs edges. The energy involved in such hydrogen bonding interactions was responsible for the higher equilibrium or binding or association constants found these systems. 
Table 3. Energetic features, thermodynamic parameters and HOMO-LUMO energy calculations for CAA, FEA and SIA and its inclusion complexes by PM3 method.

\begin{tabular}{|c|c|c|c|c|c|c|c|c|c|c|c|}
\hline Properties & CAA & FEA & SIA & $\alpha-C D$ & $\beta-C D$ & $\begin{array}{l}\text { CAA/ } \\
\alpha-\text {-CD }\end{array}$ & $\begin{array}{l}\text { CAA/ } \\
\text { B-CD }\end{array}$ & $\begin{array}{l}\text { FEA/ } \\
\alpha-C D\end{array}$ & $\begin{array}{l}\text { FEA/ } \\
\beta \text {-CD }\end{array}$ & $\begin{array}{l}\text { SIA/ } \\
\alpha-C D\end{array}$ & $\begin{array}{l}\text { SIA/ } \\
\beta \text {-CD }\end{array}$ \\
\hline $\mathrm{E}_{\text {Номо }}(\mathrm{eV})$ & -9.09 & -9.10 & -9.24 & -10.37 & -10.35 & -8.50 & -8.83 & -8.74 & -8.73 & -9.13 & -9.02 \\
\hline $\mathrm{E}_{\text {LUMO }}(\mathrm{eV})$ & -0.96 & 0.93 & -1.01 & 1.26 & $1 . .23$ & -0.48 & -0.65 & -0.92 & -1.03 & -0.88 & -0.57 \\
\hline $\begin{array}{l}\mathrm{E}_{\text {HOMO }}- \\
\mathrm{E}_{\text {LUMO }}(\mathrm{eV})\end{array}$ & -8.13 & -8.17 & -8.23 & -11.63 & -11.58 & -8.45 & -8.18 & -7.82 & -7.70 & -8.24 & -8.45 \\
\hline$\mu$ & -5.43 & -5.01 & 5.12 & -4.56 & -4.56 & -4.27 & -4.74 & -4.83 & -4.88 & -5.00 & -4.80 \\
\hline$\eta$ & -4.06 & -4.08 & 4.11 & 5.81 & 5.79 & -4.22 & -4.09 & -3.91 & -3.85 & -4.12 & -4.22 \\
\hline$\omega$ & -3.63 & -3.07 & 3.19 & 1.78 & 1.79 & -2.16 & -2.74 & -2.98 & -3.09 & -3.04 & -2.72 \\
\hline $\mathrm{S}$ & -0.25 & -0.24 & 0.24 & 0.17 & 0.17 & -0.24 & -0.24 & -0.25 & -0.26 & -0.24 & 0.23 \\
\hline $\begin{array}{l}\text { Dipole } \\
\text { moment (D) }\end{array}$ & 3.59 & 3.92 & 4.24 & 11.34 & 12.29 & 7.64 & 12.21 & 5.77 & 7.60 & 13.03 & 11.38 \\
\hline $\mathrm{E}^{*}$ & -139.5 & -132.6 & -167.2 & -1247.0 & -1457.0 & -1397.6 & \begin{tabular}{|l|}
-1605.4 \\
\end{tabular} & $\mid-1392.5$ & -1606.6 & -1420.9 & 1633.2 \\
\hline$\Delta \mathrm{E}^{*}$ & & & & & & -15.5 & $\begin{array}{l}-15.27 \\
\end{array}$ & -17.3 & \begin{tabular}{|l|}
-23.37 \\
\end{tabular} & -11.1 & -15.37 \\
\hline $\mathrm{G}^{*}$ & -65.24 & -42.77 & -60.69 & -676.36 & -789.52 & -733 & -848.4 & -709.1 & -831.5 & -719.12 & -837.10 \\
\hline$\Delta \mathrm{G}^{*}$ & & & & & & 15.6 & \begin{tabular}{ll|}
13.36 \\
\end{tabular} & 17.04 & 7.79 & 24.94 & 20.11 \\
\hline $\mathrm{H}^{*}$ & -31.70 & -7.21 & -20.34 & -570.84 & -667.55 & -610 & -706.9 & -587.9 & -689.6 & -595.27 & \begin{tabular}{|l|l|}
-692.67 \\
\end{tabular} \\
\hline$\Delta \mathrm{H}^{*}$ & & & & & & -1.48 & -3.06 & -3.87 & -10.25 & 1.89 & -0.19 \\
\hline $\mathrm{S} * *$ & 1.12 & 1.19 & 0.13 & 0.353 & 0.409 & 4.11 & 4.74 & 4.06 & 4.76 & 4.16 & 4.86 \\
\hline$\Delta \mathrm{S}^{* *}$ & & & & & & 2.64 & 3.22 & 2.52 & 3.17 & 3.68 & 4.34 \\
\hline ZPVE* & 99.37 & 116.2 & 135.79 & 635.09 & 740.56 & 736.86 & 840.70 & 754.1 & 858.9 & 773.9 & 873.4 \\
\hline
\end{tabular}

The energy change accompanying the formation of the 1:1 HCA/CD complexes can be calculated by following equation

$$
\Delta \mathrm{E}=\mathrm{E}_{\text {complex }}-\left(\mathrm{E}_{\mathrm{CD}}+\mathrm{E}_{\text {drug }}\right)
$$

where $\mathrm{E}_{\text {complex }}, \mathrm{E}_{\mathrm{HCA}}, \mathrm{E}_{\mathrm{CD}}$ are the energies of the complexes, the free (HCA) guest and free $\alpha-\mathrm{CD}$ and $\beta-\mathrm{CD}$ (host) respectively. These provide quantitative measures of the interaction forces driving the complexation process. The most stable complex among all the configurations corresponds to the greatest negative value of $\Delta \mathrm{E}$. The energy of formation values and the changes in the energies of the host (CDs) and the guest (CAA, FEA and SIA) indicated favourable formation and increased stability of complexes. Moreover, it can be seen that the mutual host-guest interactions $\left(\mathrm{E}_{\text {complex }}\right)$ contribute greatly to $\Delta \mathrm{E}$ and are crucial in determining the stability complexes $[35,36]$. The results reveal that the energy of these complexes was consistently lower than the energy sum of isolated host and guest. The binding energy of CAA $/ \alpha-C D$ and SIA $/ \beta-C D$ was $-8.45 \mathrm{~kJ} \mathrm{~mol}^{-1}$ lower than that of other inclusion complexes. Thus from the above results, it appears that amongst the six inclusion complexes the above two are more stable. 


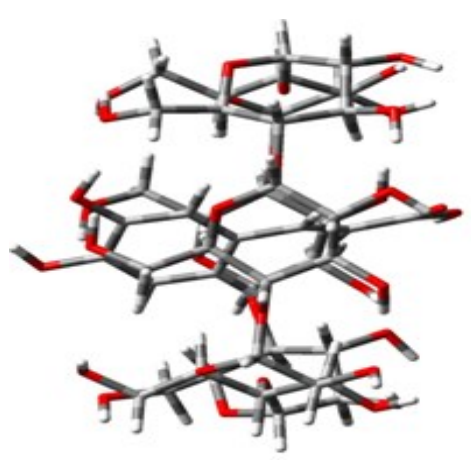

(a)
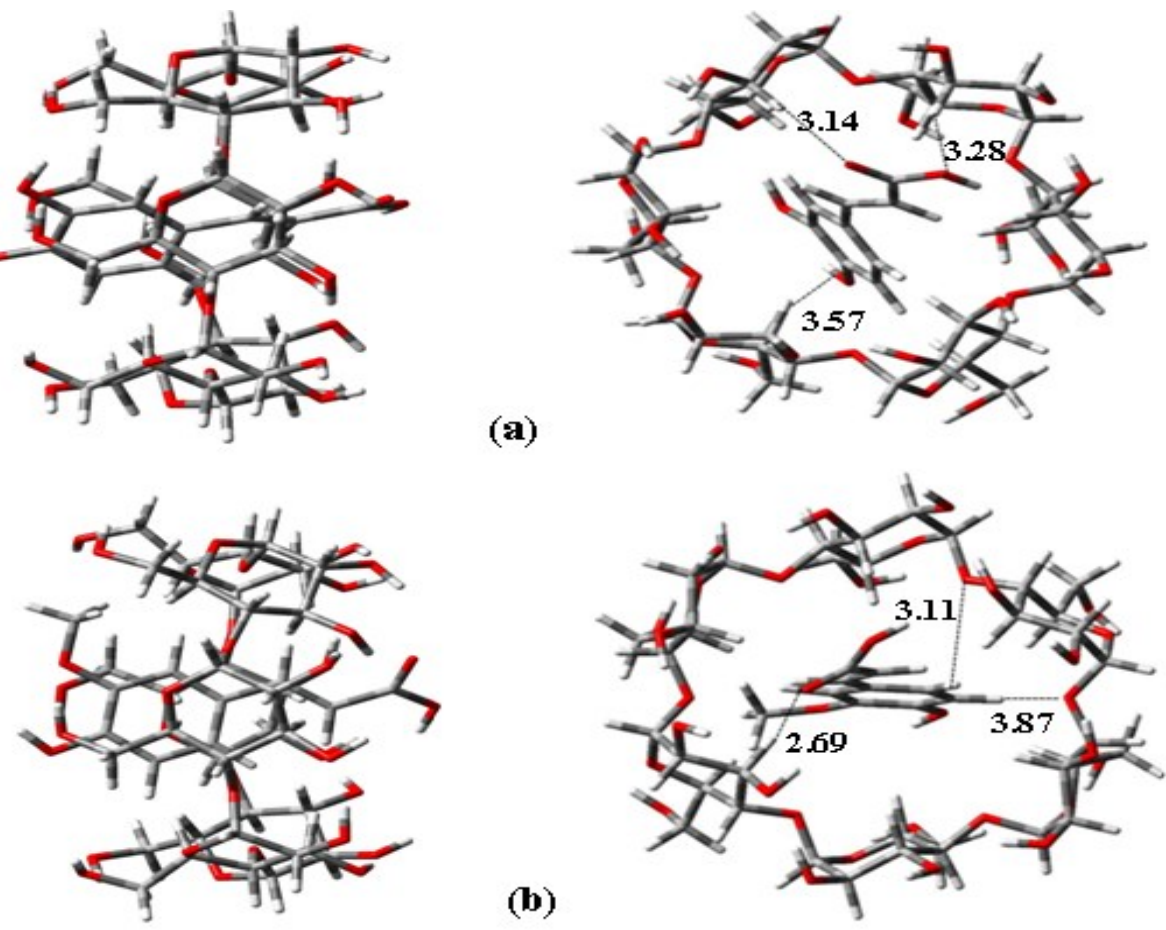

(b)
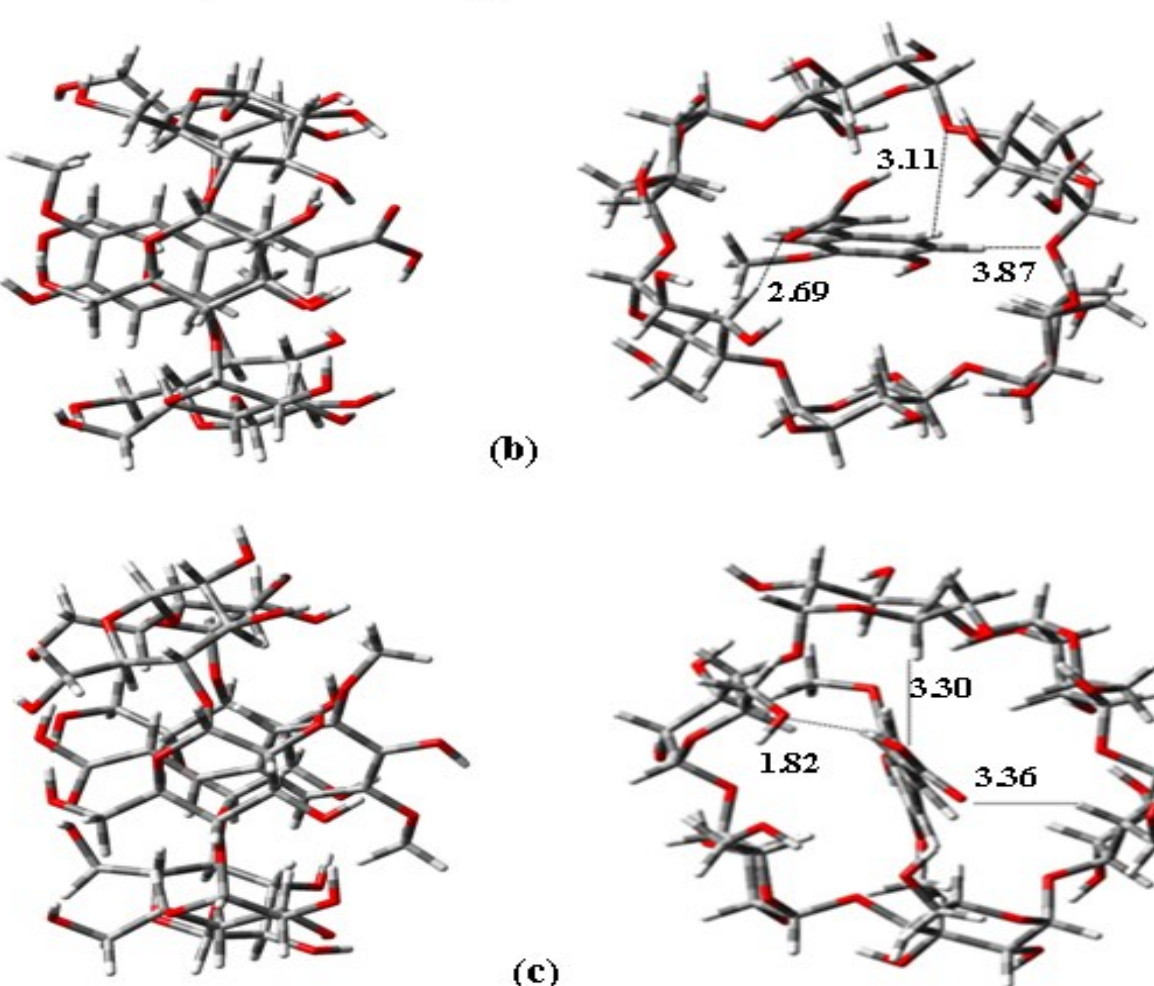

(c)

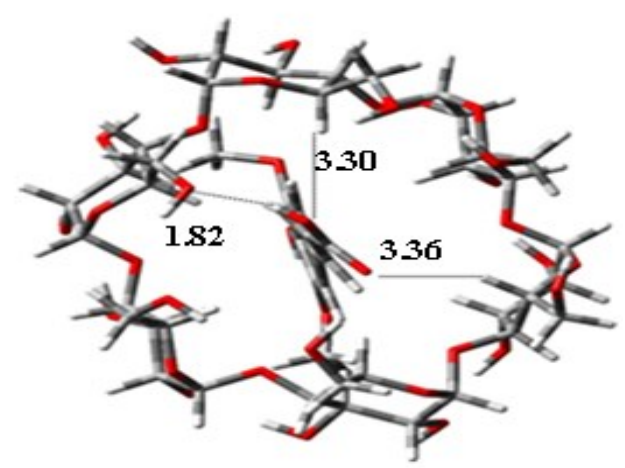

Figure 6. Side views and upper views of PM3 optimized structures of (a) CAA, (b) FEA and (c) SIA: $\alpha-C D$ inclusion complex.

The polarity of the CDs cavity was greatly decreased after the hydrophobic guest enters into the CDs cavity. As listed in Table 3, the dipole moment of SIA $\alpha-C D$ was $13.03 \mathrm{D}$ which is higher than that of native $\alpha-C D$ and $\beta-C D$. The remaining inclusion complexes are lower than that of native $C D$ values which means that the dipole moment of the complex have a closed relation with the polarity of the guest molecules [36].

$\mathrm{E}_{\mathrm{LUMO}}-\mathrm{E}_{\mathrm{HOMO}}$ gap is an important stability index and chemicals with larger $\left(\mathrm{E}_{\mathrm{LUMO}}-\mathrm{E}_{\mathrm{HOMO}}\right)$ values tend to have higher stability [37] (Table 3). Therefore, with the increase of the (E $\mathrm{LumO}^{-}$ $\left.\mathrm{E}_{\mathrm{HOMO}}\right)$ gap, the complexes formed are more stable, which agrees with the calculated results of the stability energies. It can also be seen that the $\left(\mathrm{E}_{\mathrm{LuMO}}-\mathrm{E}_{\mathrm{HOMO}}\right)$ gap of $\mathrm{CAA} / \alpha-\mathrm{CD}$ and $\mathrm{SIA} / \beta-\mathrm{CD}$ was $-8.45 \mathrm{~kJ} \mathrm{~mol}^{-1}$ lower than that of other inclusion complexes. FEA $/ \beta-\mathrm{CD}$ inclusion complex was significantly more stable than the other three complexes by an energy difference of $23.37 \mathrm{eV}$. This results shows that FEA/ $\beta-C D$ complex is more stable than other complexes.

The statistical thermodynamic calculations were carried out at $1 \mathrm{~atm}$ pressure and $298.15 \mathrm{~K}$ temperature in vacuum and the results were listed in Table 3. According to the experimental study, the $\Delta \mathrm{G}$ for the all the inclusion process was found to be positive. The positive Gibbs free energy changes for all the inclusion complexes imply that the process are non spontaneous at the room temperature, although all the complexation reactions were exothermic judged from the negative enthalpy changes. The $\Delta \mathrm{G}$ values are different from the calculated value. This discrepancy can be 
attributed, in part to the neglect the solvent effects in computational calculation as well as the hydrophobic effect that involves a gain in entropy due to the assimilation of the solvation water molecules by the medium after the inclusion takes place $[38,39]$. The step of inclusion process should be more important in the complexation of CD with the hydrophobic substrate. Therefore, the Gibbs free energy obtained from static methods (as applied here) has no absolute meaning and should be considered only in a relative way.

The very small negative $\Delta \mathrm{H}$ values indicated that the inclusion formations of the HCAs with CDs were an exothermic and enthalpy-driven $\Delta \mathrm{H}>\Delta \mathrm{S}$. It should be noted that $\Delta \mathrm{H}$ and $\Delta \mathrm{S}$ values contain contributions from (i) release of cavity found water, (ii) partial destruction of hydration shells of the reagents, (iii) non covalent interactions (van der Waals, hydrophobic and electrostatic interactions as well as hydrogen bonding and (iv) hydration of the complexes. All these process should be taken into account while discussing thermodynamic parameters of complex formation. It has been reported that the entropy of complexation depends on both the insertion of the drugs molecule and the concurrent displacement of water molecules that are trapped within the cyclodextrin cavity. Experimental results from X-ray [40] and neutron diffraction [41] as well as theoretical studies [42] have indicated that there are seven water molecules, on average, within the cyclodextrin cavity when in solution.

The enthalpy changes for the inclusion complexation of FEA/ $\beta-C D\left(-23.03 \mathrm{~kJ} \mathrm{~mol}^{-1}\right)$ was more negative than that of other inclusion complexes, which was surely attributed to the more tightly van der Waals interactions between CD and guest [43]. Thus, we can conclude that the effect of the acrylic group upon the complexation is to strengthen the van der Waals interactions. The negative enthalpy changes together with the negative entropy changes suggest that both the inclusion processes are enthalpy-driven processes in nature.

Table 4 presents the interesting bond distances, bond angles and the most interesting dihedral angles of the guests before and after complexation in $\alpha-C D$ and $\beta-C D$ obtained from from the most stable structure (Fig. 6). It was evident that in CD, the geometry of these guests was slightly altered. The alterations were significant in dihedral angles, which indicated that the drugs adopted a specific conformation to form a stable complex. Table 4 and Fig. 6 shows the intermolecular hydrogen bonding distance between the above groups with $\mathrm{CD}$ oxygens of the glucosidic bridges are greater than $3.0 \AA$ conformed the guests should not bind to the oxygens of the glucosidic bridges, as such an interaction was observed for structure. Considering the shape and dimensions of the host, the guests may not be completely embedded into the CD cavity. Since the vertical distance and length of the guests were greater than the dimensions of the host, the guest molecules cannot be fully present inside of the CD cavity. Further, the optimized theoretical structure of the guest/CD inclusion complexes also confirmed the guest molecules are partially included present in the CD cavity.

\section{Conclusion}

Encapsulation of CAA, FEA and SIA with $\alpha-\mathrm{CD}$ and $\beta-\mathrm{CD}$ has been studied by absorption, emission, time correlated single photon counting spectroscopy and molecular modeling. The CAA, FEA and SIA guests shows dual emission in the CD solution. The normal Stokes shifted bands originated from the locally excited state and the large Stokes shifted bands due to the emission from ICT state were observed. The ratio of the ICT emission to the normal emission increased with $\alpha-$ $\mathrm{CD}$ and $\beta-\mathrm{CD}$ concentration. CD studies indicates (i) HCAs forms 1:1 inclusion complex and (ii) acrylic group present in the interior part of the $\mathrm{CD}$ cavity and $\mathrm{OH} / \mathrm{OCH} 3$ groups present in the upper part of the $\mathrm{CD}$ cavity. $\mathrm{pH}$ studies suggest proton transfer reactions follow the same trend in these molecules. The positive free energy change $(\Delta G)$ suggested that all these inclusion process were not spontaneous. The van der Waals and hydrophobic interactions were the driving force for the inclusion process and also responsible for the complexes stability. The statistical thermodynamic calculations suggested that these complex processes are enthalpically favorable in nature. 
Table 4. Geometrical parameters of CAA, FEA and SIA before and after inclusion with $\alpha-\mathrm{CD}$ and $\beta-C D$ for the most stable inclusion complexes.

\begin{tabular}{|c|c|c|c|c|c|c|c|c|c|c|c|}
\hline & CAA & $\begin{array}{l}\text { CAA/ } \\
\alpha-C D\end{array}$ & $\begin{array}{l}\text { CAA/ } \\
\beta-C D\end{array}$ & & FEA & $\begin{array}{l}\text { FEA/ } \\
\alpha-C D\end{array}$ & $\begin{array}{l}\text { FEA/ } \\
\beta-C D\end{array}$ & & SIA & $\begin{array}{r}\text { SIA/ } \\
\alpha-C D\end{array}$ & $\begin{array}{l}\text { SIA/ } \\
\beta-C D\end{array}$ \\
\hline \multicolumn{12}{|l|}{$\begin{array}{l}\text { Bond length } \\
(\AA)\end{array}$} \\
\hline $\mathrm{H}_{3}-\mathrm{O}_{4}$ & 8.49 & 8.51 & 8.49 & $\mathrm{H}_{3}-\mathrm{O}_{3}$ & 8.97 & 8.93 & 8.95 & $\mathrm{H}_{2}-\mathrm{H}_{6}$ & 9.17 & 9.41 & 9.32 \\
\hline $\mathrm{H}_{4}-\mathrm{H}_{2}$ & 5.28 & 5.30 & 5.29 & $\mathrm{H}_{2}-\mathrm{H}_{8}$ & 6.50 & 6.52 & 6.48 & $\mathrm{H}_{8}-\mathrm{H}_{11}$ & 8.12 & 8.29 & 8.18 \\
\hline $\mathrm{H}_{4}-\mathrm{H}_{5}$ & 2.49 & 2.32 & 2.47 & $\mathrm{H}_{3}-\mathrm{H}_{4}$ & 1.79 & 1.80 & 1.79 & $\mathrm{H}_{1}-\mathrm{H}_{11}$ & 1.83 & 2.18 & 2.29 \\
\hline $\mathrm{H}_{6}-\mathrm{O}_{3}$ & 2.69 & 2.67 & 2.83 & $\mathrm{C}_{7}-\mathrm{O}_{3}$ & 2.94 & 2.98 & 2.92 & $\mathrm{C}_{7}-\mathrm{O}_{4}$ & 2.46 & 2.42 & 2.41 \\
\hline \multicolumn{12}{|l|}{$\begin{array}{l}\text { Bond angle } \\
\left({ }^{\circ}\right)\end{array}$} \\
\hline $\mathrm{O}_{1}-\mathrm{C}_{3}-\mathrm{C}_{4}$ & 116.8 & 124.4 & 116.4 & $\begin{array}{l}\mathrm{O}_{1}-\mathrm{C}_{3^{-}} \\
\mathrm{C}_{4} \\
\end{array}$ & 116.2 & 115.9 & 115 & $\begin{array}{l}\mathrm{O}_{1}-\mathrm{C}_{3}- \\
\mathrm{C}_{4} \\
\end{array}$ & 118.1 & 114.8 & 118.8 \\
\hline $\mathrm{H}_{2}-\mathrm{O}_{1}-\mathrm{C}_{3}$ & 107.5 & 108.6 & 108.4 & $\begin{array}{l}\mathrm{H}_{2}-\mathrm{O}_{1^{-}} \\
\mathrm{C}_{3}\end{array}$ & 94.8 & 96.81 & 100.3 & $\begin{array}{l}\mathrm{C}_{4}-\mathrm{O}_{2^{-}} \\
\mathrm{H}_{2}\end{array}$ & 108.3 & 108.5 & 108.1 \\
\hline $\mathrm{O}_{3}-\mathrm{C}_{9}-\mathrm{O}_{4}$ & 110.4 & 110.5 & 110.7 & $\begin{array}{l}\mathrm{O}_{3}-\mathrm{C}_{9^{-}} \\
\mathrm{O}_{4}\end{array}$ & 110.4 & 111.2 & 110.2 & $\begin{array}{l}\mathrm{O}_{4^{-}} \mathrm{C}_{9^{-}} \\
\mathrm{O}_{5} \\
\end{array}$ & 110.5 & 110.2 & 119.6 \\
\hline $\mathrm{C}_{1}-\mathrm{C}_{7}-\mathrm{C}_{8}$ & 122.6 & 123.2 & 122.9 & 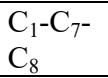 & 122.6 & 122.5 & 123.7 & $\begin{array}{l}\mathrm{C}_{7^{-}} \mathrm{C}_{8^{-}} \\
\mathrm{C}_{9} \\
\end{array}$ & 121.7 & 120.4 & 122.1 \\
\hline \multicolumn{12}{|l|}{$\begin{array}{l}\text { Dihedral } \\
\text { angle }\left({ }^{\circ}\right)\end{array}$} \\
\hline $\mathrm{H}_{2}-\mathrm{O}_{1}-\mathrm{C}_{3}-\mathrm{C}_{4}$ & 179.8 & 0.288 & 175.5 & $\begin{array}{l}\mathrm{H}_{2}-\mathrm{O}_{1^{-}} \\
\mathrm{C}_{3}-\mathrm{C}_{4}\end{array}$ & 170.3 & 167.7 & -171.3 & $\begin{array}{l}\mathrm{H}_{2}-\mathrm{O}_{1^{-}} \\
\mathrm{C}_{3}-\mathrm{C}_{2}\end{array}$ & 99.83 & 52.24 & -85.74 \\
\hline $\mathrm{H}_{3}-\mathrm{O}_{2}-\mathrm{C}_{4}-\mathrm{C}_{5}$ & -180 & 8.384 & 177.1 & $\begin{array}{l}\mathrm{H}_{5}-\mathrm{O}_{2^{-}} \\
\mathrm{C}_{4}-\mathrm{C}_{5}\end{array}$ & 176.4 & 166.2 & 165.6 & $\begin{array}{l}\mathrm{C}_{2^{-}-\mathrm{C}_{3^{-}}} \\
\mathrm{C}_{4}-\mathrm{O}_{2}\end{array}$ & 179.3 & 178.1 & -178.6 \\
\hline $\mathrm{C}_{7}-\mathrm{C}_{8}-\mathrm{C}_{9}-\mathrm{O}_{4}$ & -179.9 & 9.204 & -147.5 & $\begin{array}{l}\mathrm{C}_{7^{-}} \mathrm{C}_{8^{-}} \\
\mathrm{C}_{9^{-}}-\mathrm{O}_{4}\end{array}$ & -178.5 & 147.8 & 172.9 & $\begin{array}{l}\mathrm{C}_{1^{-}-\mathrm{C}_{7^{-}}} \\
\mathrm{C}_{8^{-}}-\mathrm{C}_{9}\end{array}$ & 179.9 & -175.7 & 179.4 \\
\hline
\end{tabular}

\section{Acknowledgement}

This work was supported by the CSIR [No. 01(2549)/12/ EMR-II] and UGC [F.No. 41351/2012 (SR)].

\section{References}

[1] M.J. Jenita, T. Mohandoss, N. Rajendiran, Spectral and molecular modeling studies on hydroxy benzaldehydes with native and modified Cyclodextrins, J. Fluores. 24 (2014) 695707.

[2] N. Rajendiran, R.K. Sankaranarayanan, Nanoscale self assembly of cyclodextrin capped 4aminobenzophenone via non-covalent interactions, J. Mol. Liq. 206 (2015) 218-227.

[3] N. Rajendiran, R.K. Sankaranarayanan, J. Saravanan, Nanochain and vesicles formed by inclusion complexation of 4, 4'-diaminobenzanilide with Cyclodextrins, J. Exper. Nanosciec. 10 (2015) 880-899.

[4] N. Rajendiran, G. Venkatesh, R.K .Sankaranarayanan, Dual fluorescence of omeprazole: Effect of solvents and pH, Phys. Chem. Liq. 52 (2014) 738-750.

[5] S. Kovendhan, G. Venkatesh, N. Rajendiran, Intramolecular charge transfer effects on 3,5diaminobenzoic acid - Effect of solvents, $\mathrm{pH}$ and $\beta$-CD, J. Ind.Chem. Society. 91 (2014) 695704.

[6] M. Jude Jenita et al., TICT effects on fast violet B and fast blue RR: Effect of HP- $\alpha-C D$ and HP- $\beta$-CDs, J.Molecular Liquids. 178 (2013) 160-167. 
[7] T. Mohandoss, J. Thulasidhasan, Dual Fluorescence of 4,4'-sulfonyldiphenol, 3,3'-dimethyl 4,4'-sulfonyldiphenol, 4,4'-sulfonyldibenzoic acid: Effects of cyclodextrin complexation, Canadian Chemical Transactions. 3 (2015) 319-332.

[8] S. Panja, S.Chakravorti, Photophysics of 4-(N,N-dimethylamino)cinnamaldehyde/ $\alpha$ cyclodextrin inclusion complex, Spectrochimica Acta Part A. 58 (2002) 113-122.

[9] P.R. Bangal, S. Panja, S.Chakravorti, Excited state photodynamics of 4-N, N-dimethylamino cinnamaldehyde:: A solvent dependent competition of TICT and intermolecular hydrogen bonding, J. Photochem. Photobiol. A Chem. 139 (2001) 5-16.

[10] S. Panja, P.R. Bangal, S.Chakravorti, Modulation of photophysics due to orientational selectivity of 4-N, N-dimethylamino cinnamaldehyde $\beta$-cyclodextrin inclusion complex in different solvents, Chem. Phys. Lett. 329 (2000) 377-385.

[11] S.Panja, S.Chakravorti, Dynamics of twisted intramolecular charge transfer process of 4-N, N-dimethylaminocinnamic acid in $\alpha$-cyclodextrin environment, Chem. Phys. Lett. 336 (2001) 57-64.

[12] S. Hamai, K. Hari, 1H-NMR Study on Inclusion Modes of 2-Chloronaphthalene and $\alpha$ Cyclodextrin in D2, Supramol. Chem. 10 (1998) 43-48.

[13] A. Nag, K. Bhattacharya, Dual luminescence of dimethylaminobenzonitrile in $\gamma$-cyclodextrin. Environmental effects on twisted intramolecular charge-transfer phenomenon, J. Chem. Soc. Faraday Trans. 86 (1990) 53-54.

[14] S. Li, W.C. Purdy, Cyclodextrins and their applications in analytical chemistry, Chem. Rev. 92 (1992) 1457-1462.

[15] K.A. Al-Hassan, The role of $\alpha$-cyclodextrin cavity size on the fluorescence of 4diethylaminobenzonitrile aqueous solution, Chem. Phys. Lett. 227 (1994) 527-532.

[16] Y. Matsushita, T. Hikida, TICT state formation in the 4'-dimethylaminoacetophenone- $\alpha$ cyclodexdtrin inclusion complex, Chem. Phys. Lett. 290 (1998) 349-354.

[17] Y.P. Sun, M.A. Fox, K.P. Johnston, Spectroscopic studies of p-(N, N-dimethylamino) benzonitrile and ethyl $\mathrm{p}$-(N, N-dimethylamino) benzoate in supercritical trifluoromethane, carbon dioxide, and ethane, J. Am. Chem. Soc. 114 (1992) 1187-1192.

[18] Y.H. Kim et al., Observation of hydrogen-bonding effects on twisted intramolecular charge transfer of $\mathrm{p}-(\mathrm{N}, \mathrm{N}-$ diethylamino)benzoic acid in aqueous cyclodextrin solution, J. Phys. Chem. 100 (1996) 15670-15676.

[19] K.S. Gould et al., Functional role of anthocyanins in the leaves of Quintinia serrata A. Cunn, J. Experimental Botany. 51 (2000) 1107-1115.

[20] M.R. Olthof et al., Chlorogenic acid and caffeic acid are absorbed in humans, J. Nutr. 131 (2001) 66-71.

[21] N.R. Prasad et al., Inhibitory effect of caffeic acid on cancer cell proliferation by oxidative mechanism in human HT-1080 fibrosarcoma cell line, Mol. Cell. Biochem. 349 (2011) 11-19.

[22] M. Hirose et al., Carcinogenicity of antioxidants BHA, caffeic acid, sesamol, 4methoxyphenol and catechol at low doses, either alone or in combination, and modulation of their effects in a rat medium-term multi-organ carcinogenesis model, Carcinogenesis. 19 (1998) 207-212.

[23] L. Elmarie van der Watt, J.C. Pretorius, Purification and identification of active antibacterial components in Carpobrotus edulis L, J. Ethnopharmacology. 76 (2001) 87-91.

[24] B. Ibtissem, C. Abdelly, S. Sfar, Antioxidant and antibacterial properties, Adv. Chem. Engin. Science. 2 (2012) 359-365. 
[25] M.G. Pierre, M. Carole, Effect of wheat variety, farming site, and bread-baking on total phenolics, Int. J. Food Science and Technology. 41 (2006) 329-332.

[26] N. Rajendiran, M.J. Jenita, Encapsulation of 4-hydroxy-3-methoxy benzoic acid and 4hydroxy-3,5-dimethoxy benzoic acid by native and modified Cyclodextrins, Spectrochimica Acta. 136 (2015) 1349-1357.

[27] M.J. Jenita, A.A.M. Prabhu, N. Rajendiran, Encapsulation of 3,5-dihydroxybenzoic acid and 3,4,5-trihydroxybenzoic acid by $\alpha$ - and $\beta$-cyclodextrins: Spectral and theoretical studies, J. Indian Chem. Soc. 91 (2014) 1711-1730.

[28] A.A.M. Prabhu, N. Rajendiran, Encapsulation of labetalol, and pseudoephedrine in $\beta-C D$ cavity: Spectral and molecular modeling studies, J. Fluorescence. 22 (2012) 1461-1474.

[29] R.K. Sankaranarayanan et al., Inclusion complexation of 3,4,5-trihydroxybenzoic acid with $\beta$ $\mathrm{CD}$ at different pH, J. Inclusion Phenomena and Macrocyclic Chemistry. 67 (2010) 461-470.

[30] RN. Rajendiran, T. Balasubramanian, Intramolecular charge transfer effects on 4-hydroxy-3methoxy benzaldehyde, Spectrochimica Acta A. 69 (2008) 822-829.

[31] T. Stalin et al., A study on inclusion complex of 1,2,3-trihydroxybenzene with $\alpha$ - and $\beta$-CD, Indian J. Chemistry. 45 (2006) 1113-1120.

[32] H.A. Benesi, J. Hildebrand, A Spectrophotometric Investigation of the Interaction of Iodine with Aromatic Hydrocarbons, J. Am. Chem. Soc. 71 (1949) 2703-2707.

[33] Y.B. Jiang, X.J. Wang, Stoichiometric-dependent intramolecular charge transfer flourescence of p-dimethylaminochalcone in $\beta$-cyclodextrin host — guest systems, J. Photochem. Photobiol. A Chem. 81 (1994) 205-209.

[34] M.R. Guzzo et al., Study of the complexation of fisetin with cyclodextrins, J. Phys. Chem. A. 110 (2006) 10545-10551.

[35] S. Chaudhuri, S. Chakraborty, P.K. Sengupta, Encapsulation of serotonin in $\beta$-cyclodextrin nano-cavities: Fluorescence spectroscopic and molecular modeling studies, J. Mol. Struct. 975 (2010) 160.

[36] En-Cui Yang et al., Semi-empirical PM3 study upon the complexation of $\beta$-cyclodextrin with 4,4'-benzidine and o-tolidine, J. Mol. Struct. Theochem. 712 (2004) 75-79.

[37] M. Karelson, V.S. Lobanov, R. Katritzky, Quantum-Chemical Descriptors in QSAR/QSPR Studies, Chem. Rev. 96 (1996) 1027-1044.

[38] W. Saenger, Cyclodextrin inclusion compounds in research and industry, Angew Chem. Int. Ed. Engl. 19 (1980)344 -362.

[39] C. Yan et al., A quantum-mechanical study on the complexation of $\beta$-cyclodextrin with quercetin, J. Mol. Struct. Theochem. 764 (2006)95-100.

[40] K. Linderner, W. Saenger, Crystal and molecular structure of cyclohepta-amylose dodecahydrate, Carbohydr. Res. 99 (1982) 103-115.

[41] T. Steiner, G. Koellner, Crystalline beta-cyclodextrin hydrate at various humidities: fast, continuous, and reversible dehydration studied by X-ray diffraction, J. Am. Chem. Soc. 116 (1994) 5122-5128.

[42] T. Heine et al., Structure and dynamics of $\beta$-cyclodextrin in aqueous solution at the densityfunctional tight binding level, J. Phys. Chem A. 111 (2007) 5648 -5655.

[43] M.V. Rekharsky, Y. Inoue, Complexation thermodynamics of Cyclodextrins, Chem. Rev. 98 (1998) 1875-1918. 